\title{
In vitro maturation, fertilization and embryo development after ultrarapid freezing of immature human oocytes
}

\author{
J. Wu ${ }^{1 *}$, L. Zhang ${ }^{1}$ and X. Wang ${ }^{1}$ \\ ${ }^{1}$ Department of Obstetrics and Gynecology, The Third Clinical Medical College, \\ Beijing Medical University, Beijing 100083, China
}

The purpose of this study was to determine the rates of maturation, fertilization and embryo development of ultrarapidly frozen immature oocytes (immature cumulus-oocyte complexes; COCs) obtained from antral follicles in ovaries of patients with chocolate ovarian cysts. The COCs were cryopreserved by a vitrification method

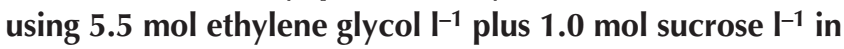
Dulbecco's PBS (DPBS). The survival, maturation and fertilization rates, and the percentage of embryos developing to the two-cell stage were $59,64,70$ and $71 \%$, respectively. No significant differences were noted in the rates of maturation, fertilization and embryo development between control and cryopreserved oocytes. Two embryos that developed from cryopreserved oocytes of the oocyte donor programme were selected for transfer into the uterus of a recipient with premature ovarian failure, after the recipient had received steroid replacement. $A$ biochemical pregnancy occurred in the recipient after embryo transfer. These results indicate that immature oocytes can survive after cryopreservation and subsequently can be cultured to mature oocytes that are capable of undergoing fertilization in vitro and developing into embryos.

\section{Introduction}

The first mammalian oocytes to be frozen and thawed successfully were those of mice and hamsters (Parkening et al., 1976; Tsunoda et al., 1976; Whittingham, 1977). Successful procedures for freezing human oocytes (Chen, 1986; Van Uem et al., 1978) were reported about 10 years later. The cryopreservation of human oocytes is an attractive alternative to embryo preservation in human in vitro fertilization (IVF) programmes and overcomes many of the ethical and legal objections to storage of human embryos (Capron et al., 1992; Perry and Schneider, 1992). Cryopreservation could be used to circumvent iatrogenic sterility after chemo- or radiotherapy. Moreover, this technique would allow potential fertility in women suffering from pathologies of the reproductive system that compromise the function of the ovaries, such as premature ovarian failure, endometriosis, cysts and pelvic infections. The use of frozen oocytes would allow the maintenance of fertility in such patients. The cryopreservation of oocytes may allow for family planning for women who delay maternity due to career demands, the absence of a partner or because of pathologies that prevent pregnancy temporarily. Finally, the use of frozen oocytes might be included in a programme of oocyte donation.

Although there are case reports of successful pregnancies after cryopreservation of unfertilized oocytes (Chen, 1986; Van Uem et al., 1978), cryopreservation of human oocytes is

Email: ji.wu@hsc.utah.edu

*Present address: Division of Urology, University of Utah School of Medicine, 50 No. Medical Drive, Salt Lake City, UT 84132, USA. still considered experimental, especially for immature human oocytes (Mandelbaum et al., 1988; Toth et al., 1994a,b; Son et al., 1996). The purpose of this study was to determine rates of maturation, fertilization and embryo development after ultrarapid freezing of human immature oocytes.

\section{Material and Methods}

\section{Collection of immature oocyte}

The Third Clinical Medical College Beijing Medical University, Beijing, China, granted ethical approval of this study. Informed consent was obtained from each patient. Ovarian tissue was obtained from 36 patients undergoing gyneacological surgery for chocolate ovarian cysts on days 9-12 after their last menstrual period. Patients ranged in age from 20 to 45 years (mean $32.0 \pm 5.8$ years) and had regular menstrual cycles ranging from 27 to 33 days. These patients had not been exposed to exogenous hormones for 3 months before the surgery. Excised ovarian tissue was washed with saline at $35^{\circ} \mathrm{C}$ to remove adhering blood clots. Immature oocytes were aspirated by puncturing the ovarian follicles (4-10 $\mathrm{mm}$ in diameter) with a 21-gauge needle attached to a syringe filled with Dulbecco's PBS (DPBS, Gibco, Beijing). Only cumulus-oocyte complexes (COCs) with a compact cumulus cell mass were included in the study. COCs were randomized and either cryopreserved or cultured as controls.

\section{Cryopreservation of immature oocyte}

Immature oocytes were frozen using a vitrification method modified from Steponkus et al. (1990) and Martino et al. (1996). The vitrification solution used was composed 


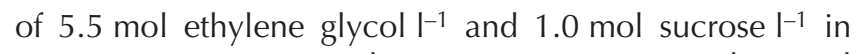
DPBS (EG 5.5). Two or three COCs were exposed to $2 \mathrm{ml}$ vitrification solution for $20 \mathrm{~s}$ and, using a pipette, were transferred in a very small volume of fluid on to electron microscope copper grids $3.05 \mathrm{~mm}$ in diameter; Electron Microscopy Sciences, Fort Washington, PA). The grids were plunged immediately into liquid nitrogen. Immature oocytes were stored in liquid nitrogen for 20-105 days.

\section{Thawing of immature oocytes}

Grids with vitrified oocytes were transferred as rapidly as possible from the liquid nitrogen into a culture dish containing $4 \mathrm{ml}$ of $0.5 \mathrm{~mol}$ sucrose $\mathrm{I}^{-1}$ at $37^{\circ} \mathrm{C}$. After $10 \mathrm{~s}$, the grids were transferred at intervals of $1 \mathrm{~min}$ into culture dishes containing sucrose solutions of $0.5,0.25,0.125$ and $0.0625 \mathrm{~mol} \mathrm{I}^{-1}$, and finally into DPBS for $5 \mathrm{~min}$. COCs were recovered from the grids in the DPBS solution using a pipette and were washed $(3 \times 5 \mathrm{~min})$ in holding medium, Ham's F10 (Flow Laboratories, Irvine) with $10 \%$ fetal cord serum.

\section{Oocyte maturation and fertilization, and embryo development}

Oocytes that showed clear bright and homogeneous cytoplasm and an intact plasma membrane and zona pellucida were considered to have survived the freezing process. These oocytes were transferred to the maturation medium after thawing. The maturation medium was Ham's F10 supplemented with 0.75 iu human menopausal gonadotrophin $\mathrm{ml}^{-1}$ (HMG; Organon, Oss) and 40\% human follicular fluid (hFF). Vitrified and control oocytes were cultured at $37^{\circ} \mathrm{C}$ in $5 \% \mathrm{CO}_{2}$ in air for $32-56 \mathrm{~h}$ in four-well culture dishes (Nunclon, Roskilde) containing the maturation medium. Control and frozen-thawed oocytes that extruded the first polar body were transferred to Ham's F10 with $10 \%$ fetal cord serum and inseminated with spermatozoa $(0.1 \mathrm{ml}$ spermatozoa at a concentration of $1 \times 10^{6} \mathrm{ml}^{-1}$ ), which had been prepared using routine washing, centrifugation and swim-up techniques (Carrell et al., 1998). The presence of two pronuclei at 16-19 $\mathrm{h}$ after insemination was used as an indication that fertilization had occurred. The ova were returned to the culture dishes for up to $144 \mathrm{~h}$ and the embryos were examined every $24 \mathrm{~h}$. The stage of embryo development was determined on the basis of the number of blastomeres. When the blastomeres of a morula began to rearrange and become organized about a central cavity (containing fluid), inner cell mass and outer cell mass, the morula was considered to be a blastocyst.

\section{Oocyte donor programme and embryo transfer}

After the pre-clinical study, an oocyte donor programme was carried out. Immature oocytes were obtained on day 12 of the menstrual cycle from a patient with chocolate ovarian cysts who was 27 years old and had a regular menstrual cycle. The oocytes were cryopreserved (sterile electron microscope copper grids, fresh liquid nitrogen and a sterile separate storage vessel were used) and fertilized with spermatozoa from the recipient's partner. Two embryos were transferred on day 18 of steroid replacement into the uterus of a 29-year-old recipient with premature ovarian failure. The exogenous steroid replacement was formulated to mimic the ovarian steroid profile during a normal 28 day menstrual cycle, and was the same as that described by Cha (1991). Urinary concentrations of hCG in the recipient were measured using an enzymeimmunoassay, 16, 18 and 20 days after embryo transfer (Kobayashi et al., 1983).

\section{Statistical analysis}

Chi-squared test was used for this statistical analysis.

\section{Results}

In the pre-clinical experiment, 157 immature oocytes were obtained from 36 patients. The mean number of immature oocytes collected per patient was $4.0 \pm 1.9$. At the time of cryopreservation, $61 \%$ of immature oocytes were at the germinal vesicle (GV) stage. Surviving oocytes had distinct intact plasma membranes (Fig. 1a)

Oocyte survival, maturation, fertilization and embryo development rates are presented (Table 1). No significant differences between control and cryopreserved oocytes were noted in rates of maturation, fertilization or embryo development (Fig. 1b,c,d). Overall, only one of 79 cryopreserved oocytes and two of 78 control oocytes reached the blastocyst stage. In the oocyte donor programme eight oocytes were collected, cryopreserved and subsequently inseminated (two-cell stage and four-cell stage). Four embryos were obtained and two were selected for transfer. A biochemical pregnancy occurred in the recipient after embryo transfer as determined by hCG in urine at days 16 , 18 and 20.

\section{Discussion}

Most attempts to freeze human oocytes have involved conventional procedures similar to those developed for freezing embryos. These protocols include slow or direct cooling of the oocytes to the seeding temperature of about $-7^{\circ} \mathrm{C}$, holding the oocytes at this temperature for a few minutes before or after seeding, and cooling them at $<1^{\circ} \mathrm{C}$ $\mathrm{min}^{-1}$ to an intermediate temperature of $-30^{\circ} \mathrm{C}$ to $-80^{\circ} \mathrm{C}$ before plunging them into liquid nitrogen (Son et al., 1996; Trounson and Kirby, 1989; Toth et al., 1994a,b). Vitrification or ultrarapid freezing of oocytes is a rapid simple technique compared with these conventional protocols. Vitrification circumvents the formation of potentially damaging intra- and extracellular ice, but cryoprotectant toxicity and osmotic injury to oocytes can occur. These effects depend on the choice and concentration of the cryoprotectant and the choice of protocol. Martino et al. (1996) succeeded in cryopreserving bovine 
Table 1. Comparison of maturation, fertilization and cleavage rates between control and cryopreserved immature human oocytes

\begin{tabular}{lcc}
\hline & Control & Cryopreserved \\
\hline $\begin{array}{l}\text { Number }(n) \\
\text { Morphological survival (\%) }\end{array}$ & 78 & 79 \\
$\begin{array}{l}\text { Maturation (\%) } \\
\text { Fertilization (\%) }\end{array}$ & $54(69)$ & $47(59)$ \\
$\begin{array}{l}\text { Two-cell embryo } \\
\text { (percentage of fertilization) }\end{array}$ & $43(80)$ & $30(64)$ \\
$\begin{array}{l}\text { Three- to eight-cell embryo } \\
\text { (percentage of two-cell embryos) }\end{array}$ & $33(77)$ & $21(70)$ \\
$\begin{array}{l}\text { Early blastocyst } \\
\text { (percentage of two-cell embryos) }\end{array}$ & $20(61)$ & $7(71)$ \\
\hline
\end{tabular}
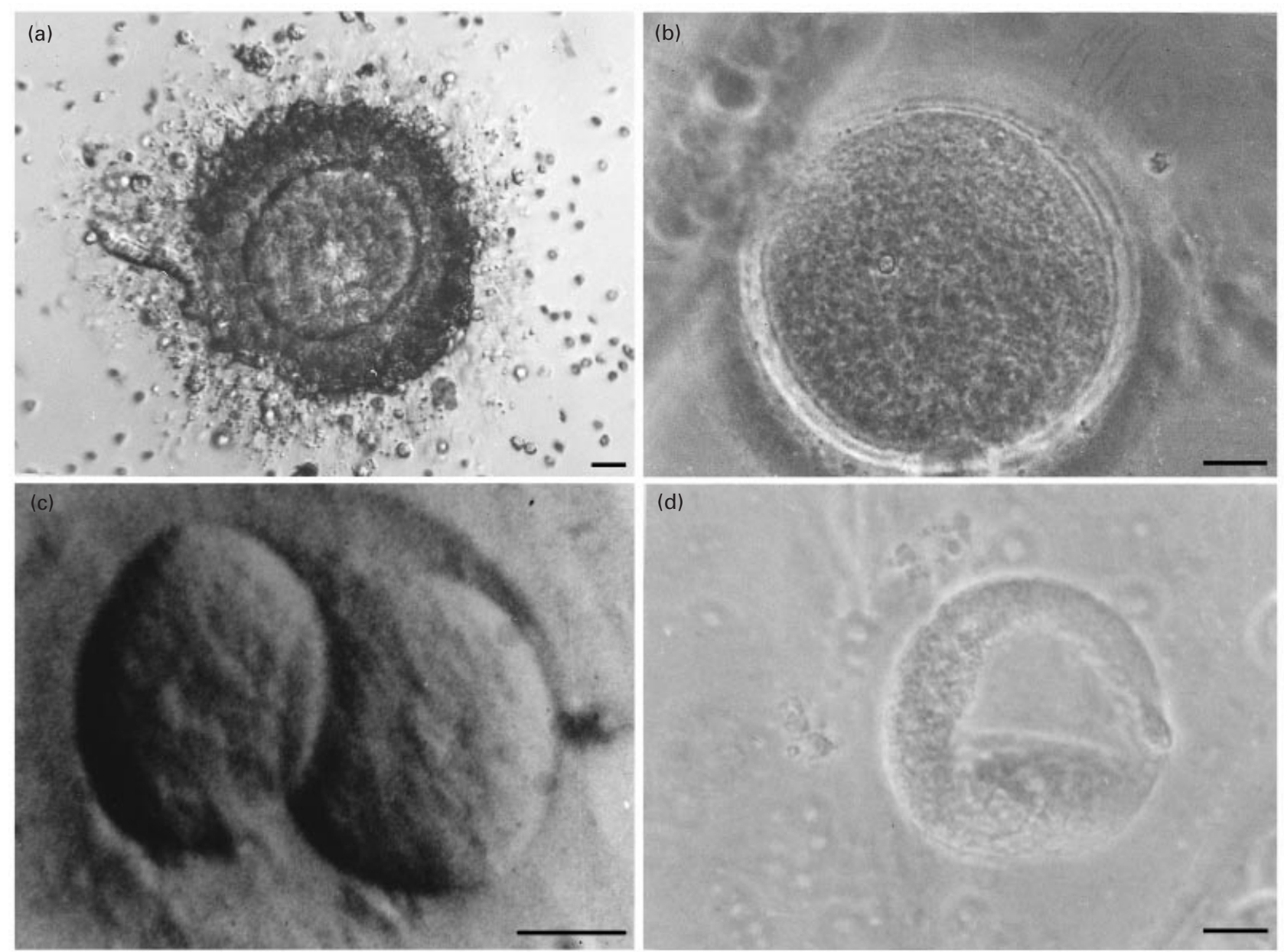

Fig. 1. Development of immature human oocytes after ultrarapid cryopreservation. (a) Immature oocyte after thawing; (b) mature oocyte after thawing and in vitro maturation; (c) Two-cell embryo and (d) early blastocyst $144 \mathrm{~h}$, respectively, after insemination of immature oocytes. Scale bars represent $25 \mu \mathrm{m}$.

oocytes using a vitrification solution containing only ethylene glycol and sucrose as cryoprotectants, and ultrarapid cooling. A significant improvement in oocyte survival was found when electron microscope grids were used as a physical support and oocytes were plunged directly into liquid nitrogen, compared with cryopreserving oocytes in straws (Martino et al. 1996). Very high cooling rates can be achieved using these grids. The estimated cooling rate for Drosophila embryos cooled on copper grids and plunged directly into liquid nitrogen was $3000^{\circ} \mathrm{C} \mathrm{min}^{-1}$, 
a rate about threefold higher than that obtained using straws (Steponkus et al., 1990). Furthermore, the use of electron microscope grids for this protocol may help rapid heat conduction from the outside to prevent the oocyte from being damaged by the solution effect of the cryoprotectant.

In the present study, 59\% of oocytes survived, $64 \%$ of the oocytes matured, $70 \%$ of the mature oocytes underwent fertilization and $71 \%$ of the fertilized oocytes cleaved. No significant differences in the rates of maturation, fertilization and embryo development were noted between control and cryopreserved oocytes.

In previous studies, $16-43 \%$ of oocytes collected from unstimulated ovaries survived freezing, and between 20 and $58 \%$ of these oocytes subsequently matured in vitro (Mandelbaum et al., 1988; Toth et al., 1994b). Son et al. (1996) reported that the survival rate of immature human oocytes after freezing and thawing was 55.1\%; and maturation, fertilization and cleavage rates were 59.3, 42.9 and 16.7 , respectively. The survival and maturation rates of immature oocytes in the present study are in agreement with the results of Son et al. (1996), but higher fertilization and embryo development rates were obtained in the present study compared with that of Son et al. (1996). This difference may be due to the use of very high cooling rates to circumvent the extreme chilling sensitivity of the oocyte.

Toth et al. (1994a) reported a maturation rate of $83.3 \%$, a fertilization rate of $56.5 \%$, and a blastocyst formation rate of $3.3 \%$ for cryopreserved immature human oocytes from stimulated IVF cycles. They suggested that the developmental capacity of immature oocytes from stimulated ovaries was higher than that of unstimulated ovaries. However, the rates of fertilization and embryo development of immature oocytes from unstimulated ovaries in the present study were similar to those from stimulated ovaries in the study of Toth et al. (1994a). In addition, it has been assumed that oocytes at prophase I collected after stimulation may be of reduced quality because immature oocytes collected after hyperstimulation demonstrate low rates of pregnancy after IVF and embryo transfer. Thus, additional work is required before conclusions can be reached regarding the developmental capacity of immature oocytes obtained from unstimulated and stimulated ovaries.

There is a risk of contamination when oocytes are brought into direct contact with liquid nitrogen. Tedder et al. (1995) reported that bone marrow samples were contaminated with hepatitis B virus during storage in liquid nitrogen. The virus was introduced into the refrigerator from a stored sample and was transferred to other samples either by failure of the integrity of the plastic sample containers used or by inadvertent contamination of the thawed sample contents from the surface of its storage bag. Procedures that avoid the risk of infection must be developed before ultrarapid oocyte cryopreservation is applied clinically.

The microtublar spindle of the mature oocyte is sensitive to temperature changes, and a potential benefit of cryopreserving oocytes at prophase $\mathrm{I}$ is that damage to the microtubule spindle may be avoided. In mice, oocytes at prophase I did not demonstrate any major abnormalities in the meiotic spindle at the second metaphase after cryopreservation and maturation (Van der Elst et al., 1992). Cryopreservation of immature oocytes at prophase I has been performed in mice (Schroeder et al., 1990; Van der Elst et al., 1992; Candy et al., 1994), rats (Pellicer et al., 1988) and humans (Mandelbaum et al., 1988; Toth et al., 1994a,b; Son et al., 1996). Candy et al. (1994) obtained live normal offspring from cryopreserved immature mouse oocytes. There are few reports of pregnancy after cryopreservation of human oocytes at prophase I (Tucker et al., 1998). Results from the current study confirm that immature human oocytes are able to survive cryopreservation and that surviving oocytes are able to mature to metaphase II, undergo fertilization in vitro and develop into early blastocysts. A biochemical pregnancy occurred in a recipient after embryo transfer. However, more detailed genetic screening and cytological analysis of the embryos obtained after maturation and fertilization of vitrified oocytes need to be undertaken before this method is applied clinically.

The authors wish to thank J. Riesen for the careful reading of the manuscript.

\section{References}

Candy CJ, Wood MJ, Whittingham DG, Merriman JA and Choudhury N (1994) Cryopreservation of immature mouse oocytes Human Reproduction 9 1738-1742

Capron AM (1992) Parenthood and frozen embryos: more than property and privacy Hastings Centre Reproduction 22 32-33

Carrell DT, Kuneck PH, Peterson CM, Hatasaka HH, Jones KP and Campbell BF (1998) A randomized, prospective analysis of five sperm preparation techniques before intrauterine insemination of husband sperm Fertility and Sterility $69122-126$

Cha KY, Koo JJ, Ko JJ, Choi DH, Han SY and Yoon TK (1991) Pregnancy after in vitro fertilization of human follicular oocytes collected from nonstimulated cycles, their culture in vitro and their transfer in a donor oocyte program Fertility and Sterility 55 109-113

Chen C (1986) Pregnancy after human oocyte cryopreservation Lancet 1 884-886

Kobayashi O, Shirotake S and Takamizawa H (1983) Studies on enzymeimmunoassay for human chorionic gonadotrophin and its clinical application Nippon Sanka Fujinka Gakkai Zasshi 35 764-772

Mandelbaum J, Junca AM, Plachot M, Alnot MO, Salar Barolx J and Alvarez A (1988) Cryopreservation of human embryos and oocytes Human Reproduction 3 117-119

Martino A, Songsasen N and Leibo SP (1996) Development into blastocysts of bovine oocytes cryopreserved by ultra-rapid cooling Biology of Reproduction 54 1059-1069

Parkening TA, Tsunoda Y and Chang MC (1976) Effects of various low temperatures, cryoprotective agents and cooling rates on the survival, fertilize ability and development of frozen-thawed mouse eggs Journal of Experimental Zoology 197 369-374

Pellicer A, Lightman A, Parmer TG, Behrman HR and DeCherney AH (1988) Morphologic and functional studies of immature rat oocytecumulus complexes after cryopreservation Fertility and Sterility $\mathbf{5 0}$ 805-810

Perry C and Schneider LK (1992) Cryopreserved embryos: who shall decide their fate? Journal of Legal Medicine 13 463-500

Schroeder AC, Champlin AK, Mabraaten LE and Eppig JJ (1990) Developmental capacity of mouse oocytes cryopreserved before and after maturation in vitro. Journal of Reproduction and Fertility 89 43-50 
Son WY, Park SE, Lee KA, Lee WS, Ko JJ, Yoon TK and Cha KY (1996) Effects of 1,2-propanediol and freezing-thawing on the in vitro developmental capacity of human immature oocytes Fertility and Sterility 66 995-999

Steponkus PL, Myers SP, Lynch DC et al. (1990) Cryopreservation of Drosophila melanogaster embryos Nature 345 170-172

Tedder RS, Zuckerman MA, Goldstone AH et al. (1995) Hepatitis B transmission from contaminated cryopreservation tank Lancet 346 $137-140$

Toth TL, Baka SG, Veeck LL, Jones HW, Jr, Muasher S and Lanzendorf SE (1994a) Fertilization and in vitro development of cryopreserved human prophase I oocytes Fertility and Sterility 61 891-894

Toth TL, Lanzendorf SE, Sandow BA, Veeck LL, Hassen WA, Hansen K and Hodgen GD (1994b) Cryopreservation of human prophase I oocytes collected from unstimulated follicles Fertility and Sterility 61 1077-1082

Trounson A and Kirby C (1989) Problems in the cryopreservation of unfertilized eggs by slow cooling in dimethyl sulfoxide Fertility and Sterility 52 778-786

Tsunoda Y, Parkening TA and Chang MC (1976) In vitro fertilization of mouse and hamster eggs after freezing and thawing Experientia 32 223-224
Tucker MJ, Wright G, Morton PC and Massey JB (1998) Birth after cryopreservation of immature oocytes with subsequent in vitro maturation Fertility and Sterility 70 578-579

Van der Elst J, Nerinckx S and Van Steirteghem AC (1992) In vitro maturation of mouse germinal vesicle-stage oocytes following cooling, exposure to cryoprotectants and ultrarapid freezing: limited effect on the morphology of the second meiotic spindle Human Reproduction 7 1440-1446

Van Uem JF, Siebzehnrubl ER, Schuh B, Koch R, Trotnow S and Lang N (1978) Birth after cryopreservation of unfertilized oocytes Lancet $\mathbf{1}$ 752-753

Whittingham DG (1977) Fertilization in vitro and development to term of unfertilized mouse oocyte previously stored at $-196^{\circ} \mathrm{C}$ Journal of Reproduction Fertility 49 89-94

Resubmitted manuscript received 18 September 2000 Accepted 9 October 2000. 